\title{
Sensor for Measuring the Atomic Fraction in Highly Dissociated Hydrogen
}

\author{
W. L. Gardner
}

Oak Ridge National Laboratory, Oak Ridge, TN 37831-8071

\section{Abstract}

Atomic hydrogen is a very important constituent for processes ranging from cleaning oxide from GaAs and annealing amorphous silicon to the deposition of diamond. Because the usual techniques for measuring atomic fraction are either expensive and cumbersome to use, or unsuitable for application to highly dissociated hydrogen, a specially designed sensor was developed. Sensor design is based on a diffusion tube with noncatalytic walls, having one end open to the atom source and a catalytic closure at the other end. The sensor is simple and inexpensive to fabricate, and determining atom density is straightforward. Sensor design also inhibits thermal runaway, which occurs when atom density is high enough to impart enough recombination energy to the non-catalytic surface to substantially raise its temperature. While recombination coefficients for such surfaces are very low near room temperature, they increase nearly exponentially with temperature unless actively cooled. With the use of a straightforward calibration scheme to determine the variation in species fraction along the diffusion tube, the atomic fraction at the tube opening is determined. Design strategy, implementation considerations, and calibration method are presented. In addition, data obtained from an atomic hydrogen source are compared to relevant published data. 


\section{DISCLAIMER}

This report was prepared as an account of work sponsored by an agency of the United States Government. Neither the United States Government nor any agency thereof, nor any of their employees, make any warranty, express or implied, or assumes any legal liability or responsibility for the accuracy, completeness, or usefulness of any information, apparatus, product, or process disclosed, or represents that its use would not infringe privately owned rights. Reference herein to any specific commercial product, process, or service by trade name, trademark, manufacturer, or otherwise does not necessarily constitute or imply its endorsement, recommendation, or favoring by the United States Government or any agency thereof. The views and opinions of authors expressed herein do not necessarily state or reflect those of the United States Government or any agency thereof. 


\section{DISCLAIMER}

Portions of this document may be illegible in electronic image products. Images are produced from the best available original document. 


\section{Introduction}

There is continuing interest in the development and characterization of sources of highly dissociated hydrogen. Atomic hydrogen is an important constituent in several emerging technologies. For example, it is effective in the removal of oxide layers on GaAs surfaces ${ }^{1}$ and in the annealing of amorphous silicon films. ${ }^{2}$ It also plays a crucial role in thin film diamond deposition. ${ }^{3}$

For characterization of atomic hydrogen sources in a production environment, a sensor is desired that is economical, easy to calibrate, and effective over a wide parameter range. A review of a variety of atom density or atom fraction measurement techniques revealed that they were either expensive and cumbersome to use or would not work properly in a highly dissociated hydrogen environment. This article introduces a sensor that avoids these shortcomings.

\section{Sensor Design}

Sensor design is based on the energy released through heterogeneous atom recombination at a highly catalytic (high recombination probability) surface. This surface forms the closure to one end of a diffusion tube having noncatalytic (very low recombination probability) walls. The other end of the sensor tube is open to the atom source.

The steady-state recombination process at the catalytic surface can be described in terms of a power balance 


$$
P=A \varepsilon_{H} \beta \Phi
$$

where $P$ is the net power input due to recombination, $A$ is the surface area, $\varepsilon_{H}$ is the bond energy of the hydrogen molecule $=7.16 \times 10^{-19} \mathrm{~J}, \beta$ is the surface accommodation coefficient, and $\Phi$ is the atom flux to the surface. The accommodation coefficient comes from the fact that only a fraction of the recombination energy is actually transferred to the surface with the rest tied up in the kinetic energy of the resultant molecule.

Figure 1 is a schematic of the sensor design. The catalytic surface is actually one end of a thermally conducting rod located a distance $L$ from the atomic hydrogen source along a cylindrical diffusion tube of radius $R$. The other end of the rod is anchored to a heat sink. The temperature difference measured by two thermocouples attached to the rod and separated by a distance $z$ is used to determine heat transfer along the rod and represents power deposited at the catalytic surface. The sensor is also designed so that no radial concentration gradients exist, and one-dimensional (1-D) solutions to the applicable diffusion equation apply.

For first-order kinetics of atom recombination in weakly dissociated hydrogen, the flux is related to the atom concentration $n$ at the surface by 4

$$
\Phi=\frac{\gamma^{\prime} v n}{4\left[1-\left(\gamma^{\prime} / 2\right)\right]}
$$

where $\gamma^{\prime}$ is the recombination coefficient defined as the fraction of incident atoms that recombine, and $v$ is the mean atom velocity. 
For a highly dissociated hydrogen source, the fact that the rate of atom removal at the catalytic surface must equal twice the rate of molecule formation leads to highly nonlinear conditions in the diffusion tube. The solution to the applicable diffusion relationship 5 is

$$
\psi=2-\left(2-\psi_{0}\right) \exp (a L)
$$

where $\psi$ is the dissociation fraction or ratio of atom to total density at the catalytic surface, and $\psi_{0}$ is the dissociation fraction at the source;

$$
a=\left[2-\left(2-\psi_{0}\right) \exp (a L)\right] / 2 \delta^{\prime} R
$$

where $\delta^{\prime}$ is a dimensionless parameter determined from sensor calibration. For a sufficiently long diffusion tube, the conditions of weak dissociation will exist at the catalytic surface. Equation 2 is then used to link Eqs. 1 and 4 to determine $\psi_{0}$

Sensor design is also influenced by the properties of diffusion tube materials. Materials such as quartz and Pyrex are good candidates with each having a room temperature $\gamma \approx 2 \times 10^{-3}$. However, $\gamma$ in each case has a nearly exponential dependence on temperature. 6 Care must be taken to keep the walls from being thermally isolated because some thermal energy is always being deposited on the wall surface. Without active cooling, thermal runaway will occur until the walls become catalytic and compromise sensor results. See the calibration section for a discussion on verifying the noncatalytic character of the walls. 


\section{Experimental}

\section{A. Apparatus}

As mentioned earlier the main elements of the sensor shown in Fig. 1 are a cooled Pyrex diffusion tube and a nickel rod to which a pair of thermocouples is attached. For the particular sensor under investigation, the diffusion tube inner diameter is $0.5 \mathrm{~cm}$ and the nickel rod diameter is $0.32 \mathrm{~cm}$. One end of the diffusion tube is open to the volume containing the atomic hydrogen, while the other end is terminated by one end of the nickel rod acting as the catalytic surface. The other end of the nickel rod is thermally anchored to the vacuum vessel but is designed to be moved axially in the Pyrex tube. As shown in Fig. 1, the Pyrex tube is actually two coaxial tubes with the annular region between them sealed at the end facing the plasma. A copper tube, thermally tied to the vacuum vessel, is then press-fit into the annular region from the other end to provide cooling. The thermocouples comprise very small diameter Type $\mathrm{K}$ wires with junctions tack-welded to the nickel rod $2.4 \mathrm{~cm}$ apart starting $0.7 \mathrm{~cm}$ from the catalytic surface. The wires are fed through a vacuum feedthrough to Analog Devices 3B37 thermocouple conditioning modules. The output from these modules is then fed to a National Instruments Model NB-MIO-16H board in a Macintosh Ilcx computer. Data acquisition software is then used for data taking. The circumference of the rod next to the catalytic surface is wrapped with Teflon ( $\gamma<$ Pyrex) tape to make that region noncatalytic and provide a good fit to the Pyrex tube wall.

The source of the atomic hydrogen is an inductively coupled plasma flow tube depicted in Fig. 2. The flow tube is made of quartz and has a water jacket for 
cooling to prevent thermal runaway of $\gamma$ that would severely degrade atomic hydrogen output. Ultra-high-purity hydrogen is fed into one end of the discharge through a flow controller with a $200 \mathrm{sccm}$ maximum flow rate. Variable radio frequency $(\mathrm{RF})$ power up to $2 \mathrm{~kW}$ is available to adjust the amount of atomic hydrogen seen by the sensor. The position of the sensor with respect to the flow tube output is also shown.

\section{B. Method}

Care was taken to reduce undesirable heat losses to a negligible level so that conduction heat flow represents power deposited by recombining hydrogen at the catalytic surface. Power to the catalytic surface is related to conduction heat flow by the usual 1-D expression

$$
P=k A_{r}\left(T_{1}-T_{2}\right) / z
$$

using known values of the thermal conductivity $k$ of nickel, the rod cross sectional area $A_{r}$, the distance between thermocouples $Z$, and the thermocouple readings $T_{1}$ and $T_{2}$. The slight linear variation of $k$ with temperature over the temperature range of interest is also accounted for through the expression

$$
k=0.941-5.65 \times 10^{-4}\left[T_{1}+T_{2}\right]\left(\mathrm{W} \mathrm{cm}^{-1}{ }^{\circ} \mathrm{C}^{-1}\right)
$$

Temperature data are taken at a fixed value of $\mathrm{L}$ of $2.0 \mathrm{~cm}(\mathrm{~L} / \mathrm{R}=8)$. This is large enough to ensure that the atom fraction is $<0.1$ at the nickel rod for the highest expected dissociation value of the source. 
The published values ${ }^{4}$ of $\gamma^{\prime}$ and $\beta$ for nickel are 0.2 and 0.82 , respectively, and are constant over the temperature range of interest $\left(<250^{\circ} \mathrm{C}\right)$. Hence, the atom density local to the nickel surface is found from Eqs. (1), (2), and (4). The atom density at the entrance of the diffusion tube is then found by solving for $\psi_{0}$ in Eq. 3.

\section{Calibration}

Calibration of this sensor to find $\delta^{\prime}$ involves using the solution to the 1-D diffusion equation with the assumption of weak dissociation that is given by 6

$$
\psi=\delta^{\prime} /\left[\delta^{\prime}+(L / R)\right]
$$

Recalling that $\psi$ is defined as $n / n_{0}$ and rearranging terms shows that the relationship of $n_{0} / n$ to $L / R$ is a straight line with slope equal to the reciprocal of $\delta^{\prime}$.

The method for calibration is to operate the atom source in a weakly dissociated mode so that Eq. 5 applies at the sensor opening. These conditions are then held constant while the rod is moved to vary L. Power to the probe tip is calculated from Eq. 4 and an examination of Eqs. 1 and 2 shows that $\left(P_{0} / P\right)=\left(n_{0} / n\right)$ where $P_{0}$ is the value of power at the sensor opening. Figure 3 is a plot of experimentally determined values of $(\mathrm{Po} / \mathrm{P})-1$ for respective values of $L / R$ at various pressures. Linear fits to the data with corresponding values determined for $\delta^{\prime}$ are also shown. 
It is important to verify the linearity of these data. A data trend showing an increasing slope with increasing $L / R$ indicates ${ }^{6}$ that the wall $\gamma$ is not $" 1$. This can be caused by surface contamination as well as by the thermal runaway effect described previously.

\section{Results and Discussion}

Once calibrated, the sensor was positioned in the diffusion tube $2 \mathrm{~cm}$ from the $\mathrm{H}$ atom source. For each of the pressures used in the calibration procedure, temperature data were taken as a function of RF power. Flow rate was maintained at $100 \mathrm{sccm}$ for the $0.25,0.5$, and 1.0 Torr cases, and at $200 \mathrm{sccm}$ for the 2.0 Torr case. A family of curves of dissociation fraction as a function of RF power was then generated as shown in Fig. 4, and the corresponding set of curves for atom density is shown in Fig. 5. A nonlinear regression model of the form

$$
y=b_{0} /\left(1+b_{1} * \exp \left(b_{2} * x\right)\right)
$$

was used to generate the solid curves in each figure to help visualize the data trends. This model was chosen because it fits the data very well and points out the fact that the atomic density at each pressure seems to asymptotically approach saturation at higher RF powers. Otherwise, no physical significance is attributed to this model.

The features of the above results are consistent with those of McCullough, et al., 7 who used a quadrupole mass spectrometer to measured hydrogen dissociation fractions produced by a microwave discharge source. The pressure and 
discharge power ranges they used are similar to those reported here. They observed both the saturation in dissociation fraction at higher discharge powers and the decrease in dissociation fraction with increasing pressure at constant power that are indicated in Fig. 4. The correspondingly higher dissociation fractions they obtained are easily explained by the very small exit nozzle used in their apparatus. This small-diameter nozzle greatly increases the residence time for dissociation of the molecular species in their discharge.

The basic agreement between this sensor and a mass spectrometer on a similar hydrogen dissociation source lends credence to the design and calibration methodologies presented above. Hence, it is believed that this sensor reasonably measures the atomic fraction in highly dissociated hydrogen over a wide range of parameters. In addition, it has the advantage over optical techniques of producing results directly without calibration to an absolute standard. It is also very economical to implement and should be much easier to implement than a mass spectrometer system. Lastly, it can be easily modified to measure dissociation fractions in oxygen, nitrogen, chlorine, and fluorine environments.

\section{Acknowledgments}

Research was sponsored by the Laboratory Directed Research and Development Program of the Oak Ridge National Laboratory, managed for the U.S. Department of Energy by Martin Marietta Energy Systems, Inc. under contract No. DE-AC05-84OR21400. 


\title{
References
}

1. A. Watanabe, M. Hata, and T. Isu, J. Cryst. Growth 111, 554 (1991).

2. Uchida, H. Kanoh, O. Sugiura, and M. Matsumura, Japan J. Appl. Phys. 29, L2171 (1990).

3. C. Angus and C. C. Hyman, Science 241, 913 (1988).

4. J. Wood, J. S. Mills, and H. Wise, J. Phys. Chem: 671462 (1963); Errata: J. Phys. Chem. 68, 3911 (1964).

5. G. Dickens, D. Schofield, and J. Walsh, Trans. Faraday Soc. 56, 225 (1960).

6. H. Wise and C. M. Ablow, J. Phys. Chem. 29, 634 (1958).

7. J. Wood and H. Wise, J. Phys. Chem. 66, 1049 (1962).

8. W. McCullough, J. Geddes, A. Donnelly, M. Liehr, M. P. Hughes, and H. B. Gilbody, Meas. Sci. Technol. 4, 79 (1993).

\section{DISCLAIMER}

\begin{abstract}
This report was prepared as an account of work sponsored by an agency of the United States Government. Neither the United States Government nor any agency thereof, nor any of their employees, makes any warranty, express or implied, or assumes any legal liability or responsibility for the accuracy, completeness, or usefulness of any information, apparatus, product, or process disclosed, or represents that its use would not infringe privately owned rights. Reference herein to any specific commercial product, process, or service by trade name, trademark, manufacturer, or otherwise does not necessarily constitute or imply its endorsement, recommendation, or favoring by the United States Government or any agency thereof. The views and opinions of authors expressed herein do not necessarily state or reflect those of the United States Government or any agency thereof.
\end{abstract}


Figure Captions

Fig. 1 A schematic of the atomic hydrogen sensor design.

Fig. 2 The atomic hydrogen flow tube source with the sensor positioned at its output.

Fig. 3 A plot of experimentally determined values of $\left(\mathrm{P}_{\mathrm{O}} / \mathrm{P}\right)-1$ for respective values of $L / R$ at various pressures. Solid lines represent linear fits to the data. Values of $\delta^{\prime}$ for each case are also shown.

Fig. 4 A plot of hydrogen dissociation fraction as a function of RF power at different pressures. Solid lines represent a nonlinear fit to the data.

Fig. 5 A plot of atomic hydrogen density data corresponding to the data plotted in Fig. 4. Again the solid lines represent a nonlinear fit to the data. 


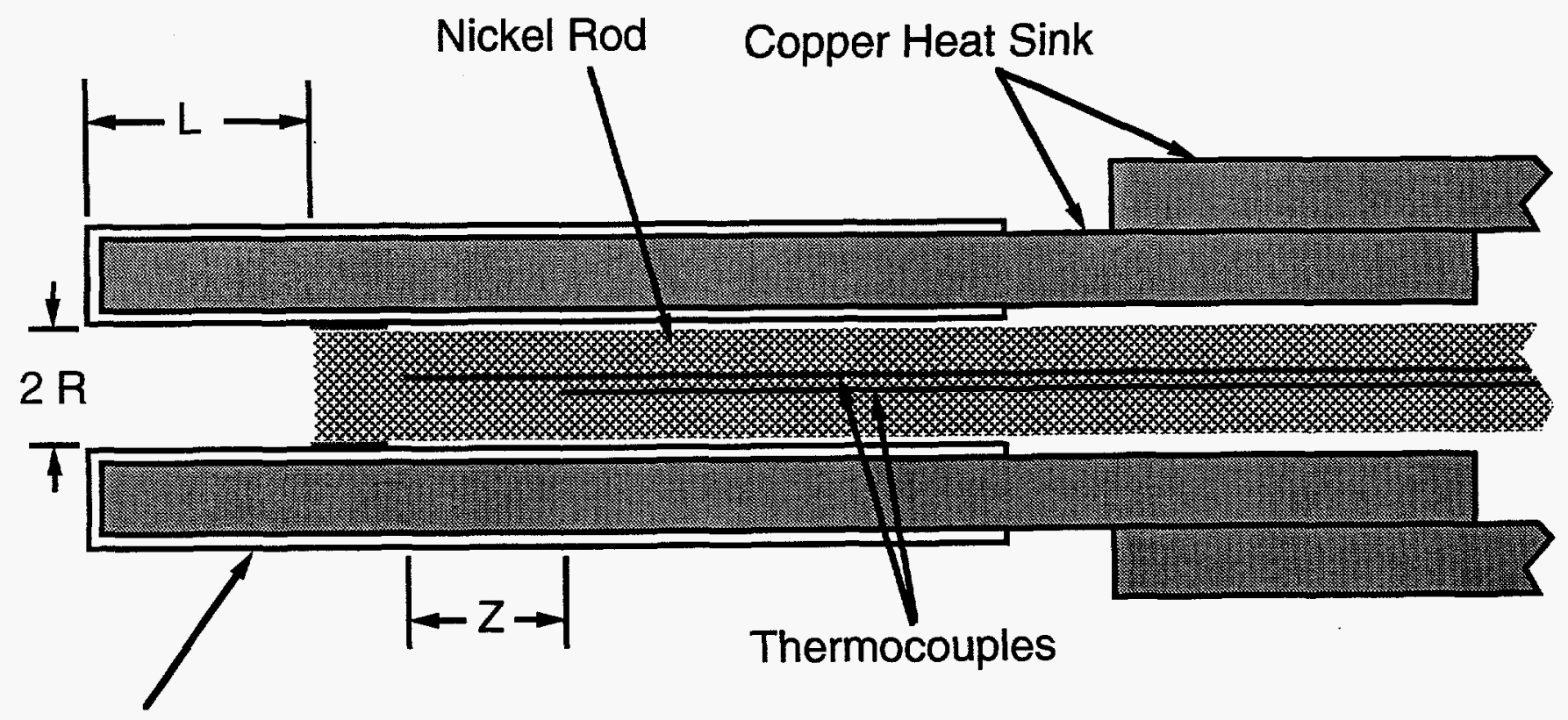

Pyrex Diffusion Tube 


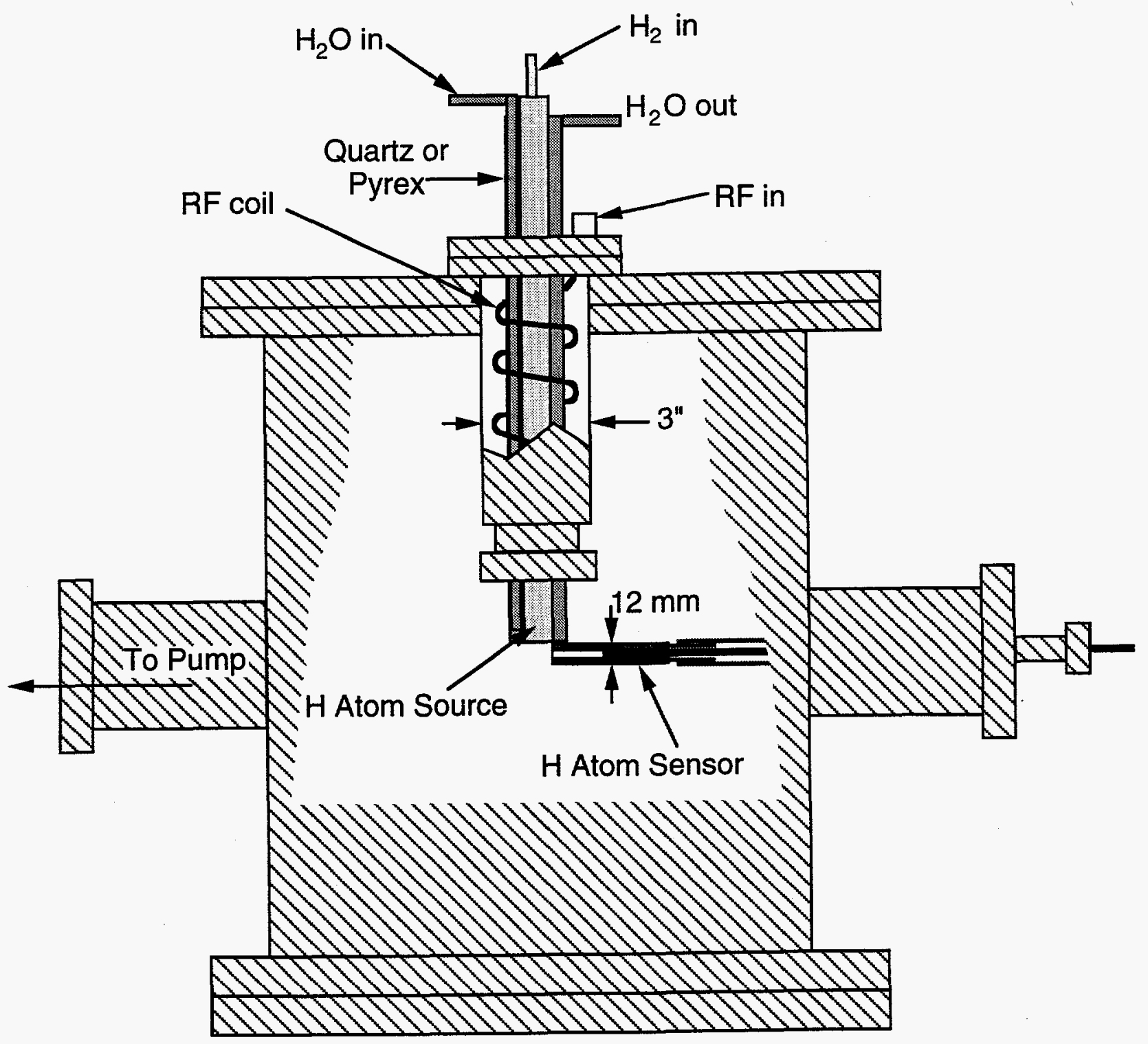




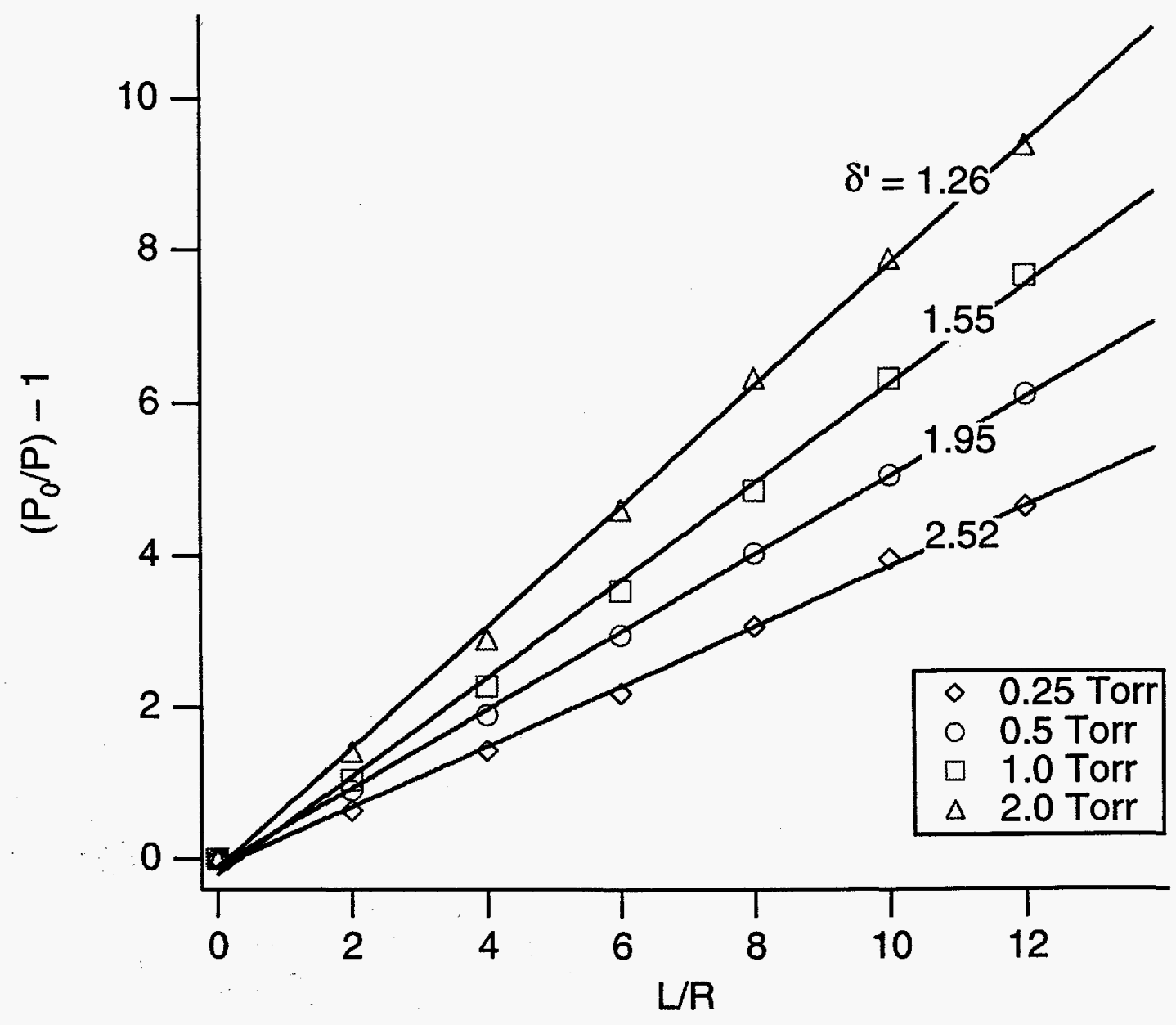




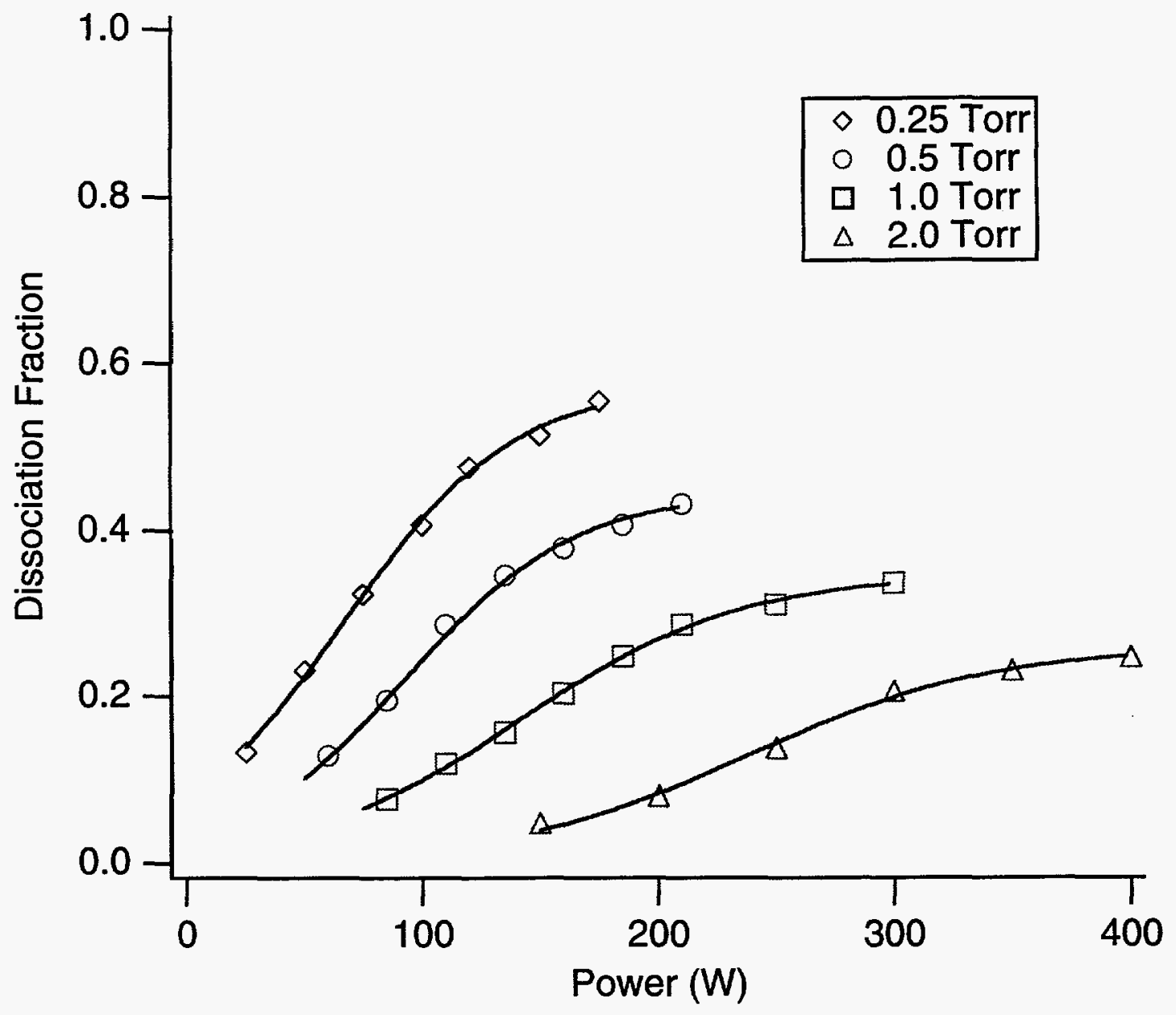




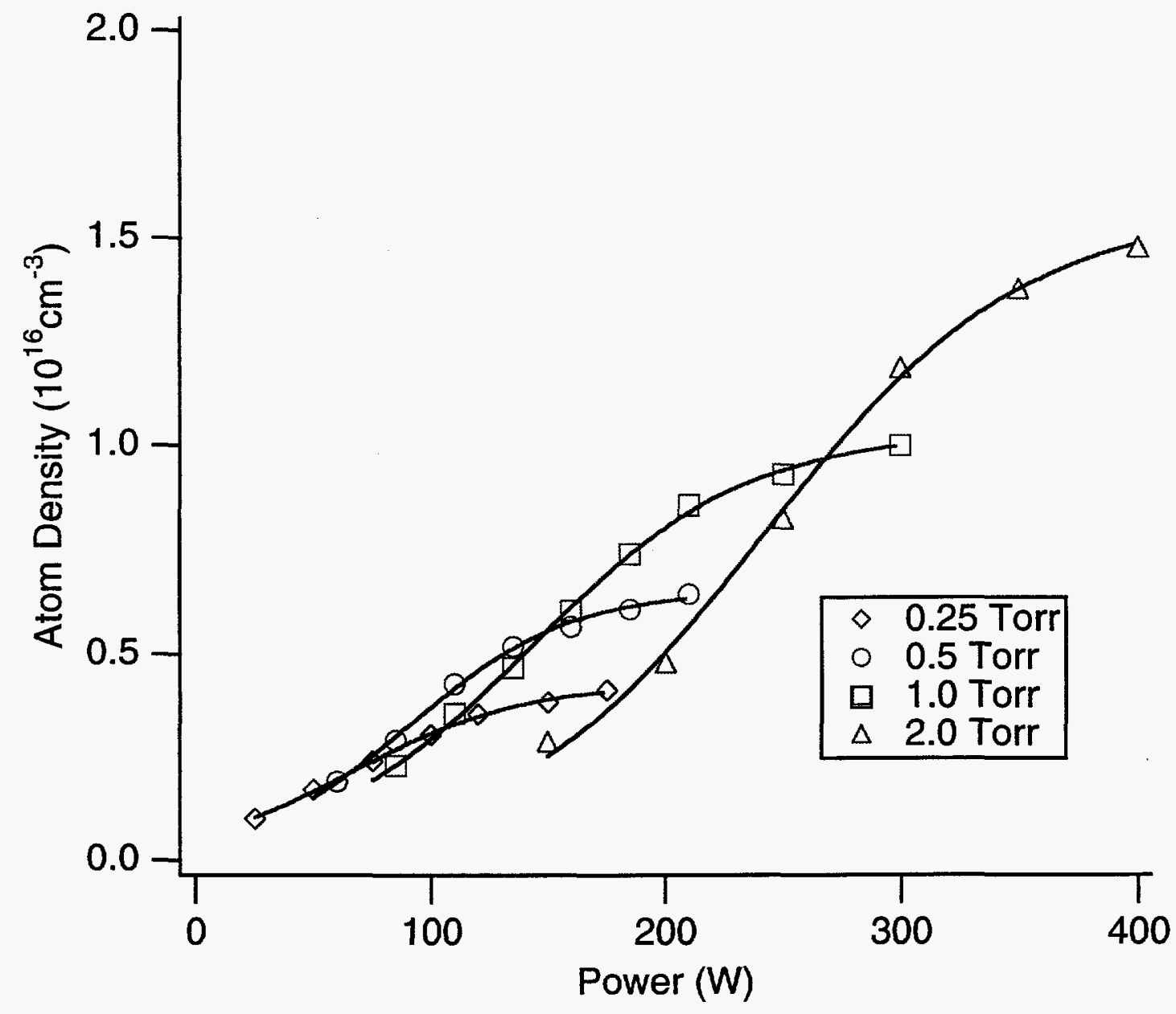

